\title{
Enhanced Transmission Performance Using Backward-Propagated Broadband ASE Pump
}

\author{
Md Asif Iqbal, Mingming Tan, and Paul Harper
}

\begin{abstract}
We propose a novel first order incoherent broadband ASE pump which can be used as a seed pump in dual order backward-pumped distributed Raman amplification. Using this broadband ASE pump mitigates the RIN transfer from the backward-propagated second order pump to the signal, and extends the reach of $10 \times 120 \mathrm{~Gb} / \mathrm{s}$ DP-QPSK WDM transmission to $7915 \mathrm{~km}$, giving a minimum of $12 \%(833 \mathrm{~km})$ increase, compared with using low RIN semiconductor laser diode and random distributed feedback (DFB) fiber laser.
\end{abstract}

Index Terms-Optical amplifiers, optical fiber communication.

\section{INTRODUCTION}

U sing distributed Raman amplification (DRA) in optical fiber communications systems reduces the signal power variation along the transmission fiber, which can provide a better balance between the amplified spontaneous emission (ASE) noise and the Kerr nonlinearities. This improves the maximum transmission reach/data capacity, compared with lumped amplification [1]-[3]. To achieve this, different Raman pumping schemes can be used. Bidirectional pumping schemes have been shown to give better performances, but there is a major problem that the use of forward (FW) pumping leads to a relative intensity noise (RIN) induced penalty which limits the performance enhancement in long-haul repeatered transmission systems [4]-[7]. Different methods of generating FW pumps have been proposed to reduce this penalty [8]-[10], but there are still limits on the FW pump power that can be used and low Raman gain efficiency in the power transfer from the pump to the signal can be low. A second technique to improve the signal power distribution is the use of higher order pumping to generate a random distributed feedback (DFB) fiber laser. The first order random DFB fiber laser generated in the transmission fiber due to the fiber Rayleigh backscattering and/or the inclusion of fiber Bragg grating (FBG) as reflectors, provides signal gain and doesn't require a specially customized $\mathrm{FW}$ pumping source [11]-[15]. However, as this is essentially bidirectionally-propagated pumping in a laser cavity, the RIN induced penalty remains difficult to mitigate. Consequently, backward (BW) pumping is preferred in long-haul transmission system to avoid the fundamental problem of RIN transfer from

Manuscript received xxxx, 2018; revised xxxx, 2018; accepted xxxx, 2018. Date of publication xxxx, 2018; date of current version xxxxx, 2018.

This work was funded by FP7 ITN programme ICONE (608099), UK EPSRC Programme Grant EP/J017582/1 and EP/L000091/1(UNLOC and PEACE). We thank C. Wang, Z. Sun, and L. Zhang for providing the FBGs, and S. Sugavanam for useful discussions (Corresponding author: M. Tan).
FW pump(s) to signal. The RIN transfer is low in BW-pumping DRA due to the averaging over longer span length and lower cut-off frequency of the RIN transfer function [7]. However, even this small amount of RIN transfer over 10-100s of $\mathrm{kHz}$ from high power and high RIN pump(s), can still limit the transmission performance, especially in long-haul repeatered systems.

In this paper, for the first time, we present a dual order BWpumping only scheme which uses an incoherent broadband first order pump. Here, the dual order BW-pumping scheme consists of a $1366 \mathrm{~nm}$ second order fiber laser and a broadband $1453 \mathrm{~nm}$ first order seed pump generated by Rayleigh scattering. This seed pump mitigates the signal RIN penalty by preventing the evolution of RIN transfer from the higher order pump to the signal. We demonstrate that, using this incoherent broadband pump provides superior long-haul transmission performance in comparison with using low RIN semiconductor pumps and random DFB fiber laser generated by backward second order pump. In a 100G DP-QPSK WDM long-haul transmission system, our proposed scheme extends the transmission reach to $7915 \mathrm{~km}$ with an $833 \mathrm{~km}$ transmission reach improvement. In addition, the use of such broadband pumping can provide a flatter gain spectrum, which reduces the number of pumps required in broadband (i.e. $\mathrm{C}+\mathrm{L}$ band) transmission [16], [17].

\section{EXPERIMENTAL SETUP OF THE PROPOSED DistRIBUTED RAMAN AMPLIFIER}

Dual order distributed Raman amplification configurations were investigated using $83.32 \mathrm{~km}$ standard single mode fiber (SSMF) as the transmission spans. Second order backwardpropagated pump at $1366 \mathrm{~nm}$ (Raman fiber laser, $\sim 7 \mathrm{~nm} 3 \mathrm{~dB}$ bandwidth) was used in combination with one of three different first order pump sources: the new broadband incoherent pump source which we have developed, low RIN semiconductor laser diodes, and random DFB fiber laser. Our broadband incoherent pump is shown in Fig. 1(a). The Rayleigh backscattered light was generated and amplified using second order BW pump over $10 \mathrm{~km}$ SSMF. The light was incoherent amplified spontaneous emission (ASE) which was centered at $1453 \mathrm{~nm}$ and similar to broadband Raman gain spectrum [18]. Then, the generated

Md. A. Iqbal, M. Tan, and P. Harper are with Aston Institute of Photonics Technologies, Aston University, Birmingham, B4 7ET, UK. (e-mail: iqbalm7@aston.ac.uk; m.tan1@aston.ac.uk; p.harper@aston.ac.uk)

Original data for this work is available through Aston Research Explorer (https://doi.org/10.17036/researchdata.aston.ac.uk.00000342). 
broadband ASE light at $1453 \mathrm{~nm}$ was fed into the $10 \mathrm{~km}$ TrueWave fiber span with higher Raman gain efficiency $(0.6 / \mathrm{W} / \mathrm{km})$ and then amplified by the residual $1366 \mathrm{~nm}$ pump from the other side of the $10 \mathrm{~km}$ SSMF. Therefore, the broadband incoherent $1453 \mathrm{~nm}$ ASE was powerful enough to be used as the first order pump after the isolator. The output power was up to $250 \mathrm{~mW}$ with $3.8 \mathrm{~W}$ second order pump power. The length of SSMF in the broadband pump generation section was optimized for efficient, stable generation of the $1453 \mathrm{~nm}$ broadband pump. If the SSMF length was increased, the threshold of generating the random laser spikes would be significantly reduced and the output power of the backscattered light would also be decreased due to the increase in fiber loss [18]. In addition, the fiber type used to generate the broadband ASE light was also optimized to enhance the broadband pump power and avoid the random lasing instability simultaneously. The inset in Fig. 1(a) shows the broadband pump power vs second order pump power using two types of fiber: $10 \mathrm{~km}$ SSMF and $2 \mathrm{~km}$ dispersion compensating fiber (DCF). The Raman gain coefficient of DCF is higher than SSMF, but the random lasing threshold was much lower for the DCF than SSMF due to the DCF having higher Rayleigh backscattering [19]. Using DCF limits the maximum outpower of broadband ASE, as the broadband ASE would become unstable spiky random fiber laser even using low pump power. Therefore, the achievable broadband pump power was decreased substantially, as the instability of random lasing in DCF became problematic.

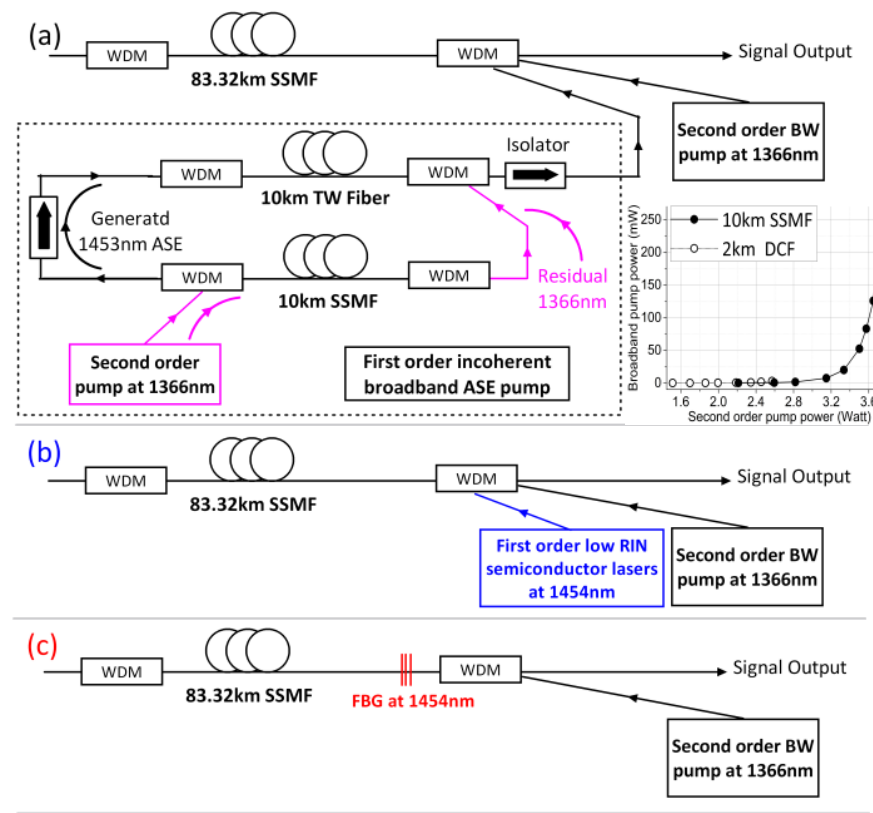

$\overline{\text { Fig. 1. Dual order distributed Raman amplification configurations using second }}$ order BW pumping and different first order seed pump; (a). Broadband incoherent ASE pump. (b). Low RIN semiconductor laser diodes. (3). Random DFB fiber laser. Inset in Fig.1(a). The generated broadband pump power versus second order pump power at $1366 \mathrm{~nm}$ using $10 \mathrm{~km}$ SSMF or $2 \mathrm{~km}$ DCF.

The semiconductor laser used as the first order pump shown in Fig. 1(b), were commercially available low RIN semiconductor laser diodes. Two $1454 \mathrm{~nm}$ lasers were combined using a polarization beam combiner (PBC), giving a maximum output power of $400 \mathrm{~mW}$. A fixed $20 \mathrm{~mW}$ first order seed power and $1 \mathrm{~W}$ second order pump power was used for both the broadband pump and semiconductor laser configurations to overcome the $16.5 \mathrm{~dB}$ loss of $83.32 \mathrm{~km} \mathrm{SSMF}$.

For the random distributed feedback fiber laser (as shown in Fig. 1(c)), a fiber Bragg grating centered at $\sim 1454 \mathrm{~nm}$ with $3 \mathrm{~dB}$ bandwidth of $0.5 \mathrm{~nm}$ and $>95 \%$ reflectivity was used at the output end of the fiber span. As the second order pump power was increased, first order random fiber laser was generated due to the resonant mode overcoming the lasing threshold in a fiber cavity [13]. This fiber laser cavity was "half-open", as the Rayleigh scattering from the fiber was an "open" distributed reflector, and a high reflectivity FBG was "closed" as the other reflector [11]. 1.1 W second order pump power was used to compensate the fiber attenuation, while the power of the random fiber laser measured in the output direction between the SSMF and the FBG was $\sim 13.6 \mathrm{~mW}$.

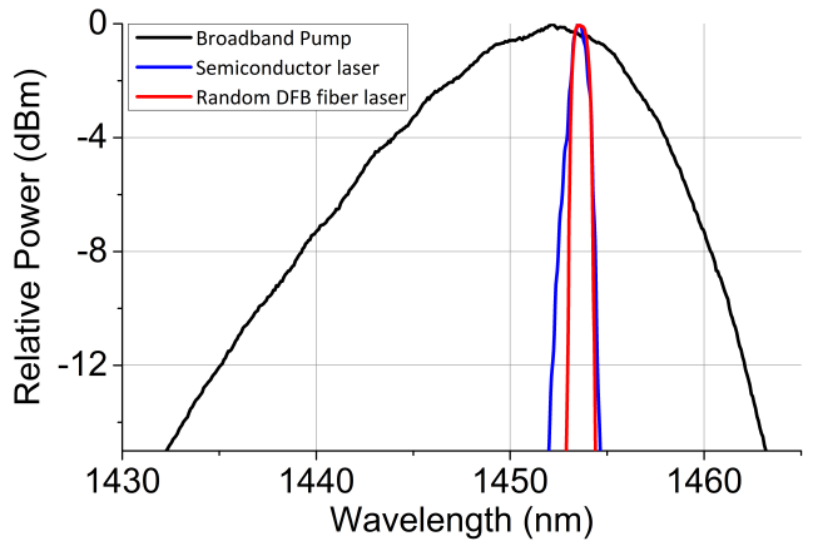

Fig. 2. Measured optical spectra of three first order pumps.

Figure 2 shows the optical spectra of the three first order pumps. The $3 \mathrm{~dB}$ bandwidth of the proposed broadband ASE pump was $\sim 12 \mathrm{~nm}$, which was >10 times wider than the semiconductor laser diode $(\sim 0.8 \mathrm{~nm})$ and random DFB fiber laser $(\sim 0.6 \mathrm{~nm})$.

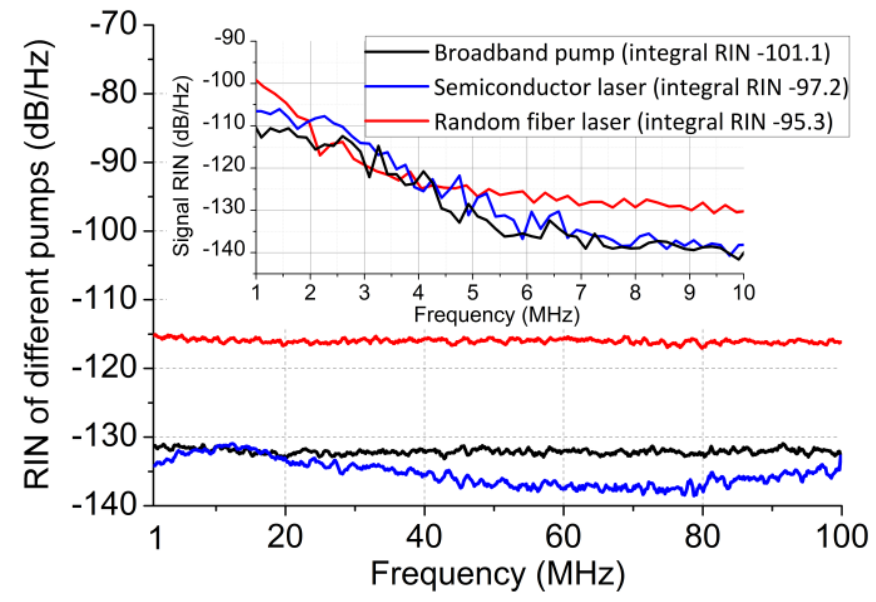

Fig. 3. Measured RIN spectra of three first order pumps. Inset: Measured signal RIN using three first order pumps in dual order BW pumping configurations.

The RIN of three first order pumps are shown in Fig. 3 ranging from 1 to $100 \mathrm{MHz}$ using the setup illustrated in [4]. The semiconductor laser showed the lowest RIN level of -134 $\mathrm{dB} / \mathrm{Hz}$ at $1 \mathrm{MHz}$. In comparison, the broadband ASE pump showed a similar RIN level of $-132 \mathrm{~dB} / \mathrm{Hz}$ at $1 \mathrm{MHz}$. For the random fiber laser, the RIN was $-115 \mathrm{~dB} / \mathrm{Hz}$ at $1 \mathrm{MHz},>17 \mathrm{~dB}$ 
higher than the other two pumps. Interestingly, both the broadband ASE pump and the random DFB fiber laser were both generated by the same type of second order pump at 1366 $\mathrm{nm}$ with the RIN of $\sim-125 \mathrm{~dB} / \mathrm{Hz}$. The broadband ASE pump was formed in a two-stage process. The first stage was effectively a Stokes shift process, and this was the reason why the optical spectra resembles a Raman frequency shift spectrum, which generated the low-power broadband ASE light. Then the ASE was used as a signal to the second stage which was essentially a $10 \mathrm{~km}$-long distributed Raman amplifier pumped by the residual $1366 \mathrm{~nm}$ pump. As the pump at 1366 $\mathrm{nm}$ was BW propagated, the signal (the ASE light) was not strongly affected by the high RIN BW pump after an unrepeatered $10 \mathrm{~km}$ span. In contrast, the random DFB fiber laser was very different. Due to the use of the high reflectivity FBG, the power transfer efficiency between the second order high RIN pump and the induced fiber laser was significantly higher, compared with the ASE generated by the Stokes shift. In addition, the fiber itself was a laser cavity, meaning that there were both laser components from either forward or backwardpropagation from the perspective of the second order pump [4]. Consequently, the second order pump efficiently transferred its RIN to the induced random fiber laser, which resulted in the high RIN of random fiber laser. In these dual-order BW pumped DRAs, the main RIN transfer to the signal came from the high RIN second order pump laser. The highest RIN transfer was obviously expected from the random DFB fiber laser. The semiconductor laser had the lowest RIN, but the narrow bandwidth profile still allowed some RIN transfer from high RIN second order pump to signal [10]. The broadband ASE pump also had similar RIN to the semiconductor laser, but the RIN from the second order pump was averaged out due to the non-degenerate four-wave mixing effect as the phase was rapidly changed over such a large bandwidth of the first order pump [20], which subsequently ensured the lowest RIN transfer to the signal. The inset in Fig.3 shows the measured signal RIN and its integral ranging from 1 to $10 \mathrm{MHz}$ using three different first order pumps in dual order BW-pumped DRAs, which confirms the analysis above.

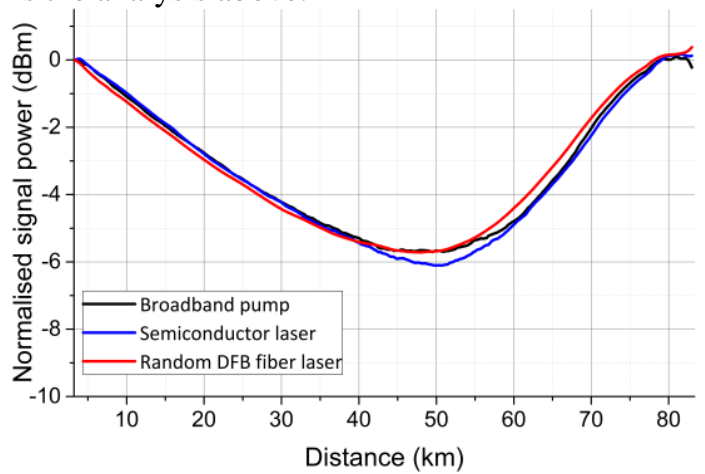

Fig. 4. Measured signal power profiles in dual order BW-pumping schemes using broadband ASE pump, semiconductor laser, or random DFB fiber laser.

Signal power profiles along the transmission fiber were experimentally measured at $1545.3 \mathrm{~nm}$ using optical timedomain reflectometry (OTDR). All the configurations gave almost the same signal power profiles with signal power variation (SPV) of $\sim 6 \mathrm{~dB}$. This was also the reason why the first order pump power for broadband ASE pump and semiconductor laser was set to be $20 \mathrm{~mW}$, otherwise it would result in different signal power profiles. The same signal power profiles mean that with the same input signal launch power, all configurations had the same ASE noise performances and nonlinearity impairments, i.e. they would have the same tradeoff between the ASE noise and nonlinearity impairments [10].

\section{TRANSMISSION RESULTS AND DISCUSSIONS}

A recirculating loop experiment was conducted to evaluate the transmission performances in the long-haul repeatered system illustrated in Fig. 5. Ten DFB lasers spaced at $100 \mathrm{GHz}$ (from $1542.94 \mathrm{~nm}$ to $1550.12 \mathrm{~nm}$ ) and a "channel under test" (a $100 \mathrm{kHz}$ linewidth tunable laser) were combined to give a 10channel WDM grid. The output lasers were QPSK modulated at $30 \mathrm{GBaud}$ and polarization-multiplexed to give $10 \times 120 \mathrm{~Gb} / \mathrm{s}$ DP-QPSK signal. The Raman amplified link in the recirculating loop was $83.32 \mathrm{~km}$ SSMF ( 16.5 dB attenuation) including pump signal combiners ( $\sim 1.1 \mathrm{~dB}$ attenuation). A dynamic gain flattening filter (GFF) was included. The total loop specific loss was $\sim 12 \mathrm{~dB}$ from the $3 \mathrm{~dB}$ coupler, the GFF, the acousto-optic modulator (AOM), and pump signal combiners, and a dualstage EDFA was to compensate this. The output signal was demultiplexed by a tunable filter and amplified into the standard polarization-diverse coherent receiver. Standard DSP algorithm was used offline for the linear impairments. Q factors were calculated from the bit error rate (BER), and averaged over 2 million bits.

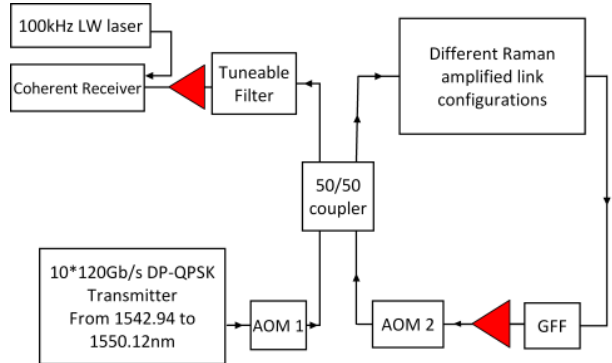

Fig. 5. Schematic diagram of long-haul repeatered transmission system using the proposed distributed Raman amplifiers demonstrated in Fig. 1.

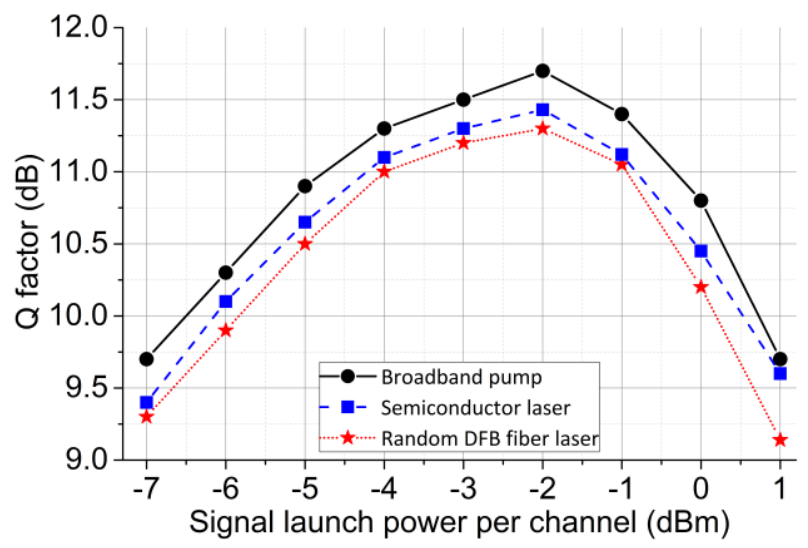

Fig. 6. Measured $1545.3 \mathrm{~nm}$ central channel Q factors versus signal launch power at $3333 \mathrm{~km}$ using broadband pump, semiconductor laser, and random DFB fiber laser in dual order BW pumping configurations. 
Figure 6 shows the experimental Q factors versus signal launch power per channel at $3333 \mathrm{~km}$ for all configurations. As indicated from the signal power profiles (Fig. 4), similar Q factors should be expected from all the configurations. However, this was not the case. In dual order backward pumped configuration, using the broadband ASE pump as the seed gave the best $\mathrm{Q}$ factor of $11.7 \mathrm{~dB}, 0.3 \mathrm{~dB}$ better than using semiconductor laser, and $0.4 \mathrm{~dB}$ higher than random DFB fiber laser configuration. In particular, the improvement occurred at all the signal power levels in both linear and nonlinear regimes. In addition, all the configurations had the same optimum launch powers. This indicates that neither ASE noise nor nonlinearity was responsible for the performance degradation when using semiconductor laser or random fiber laser. More importantly, the RIN transfer from the high RIN second order backwardpropagated pump to the signal via the first order seed caused Q factor penalty in long-haul repeatered transmission system [4]. However, compared with forward-pumping schemes [4]-[7], this penalty was substantially smaller.

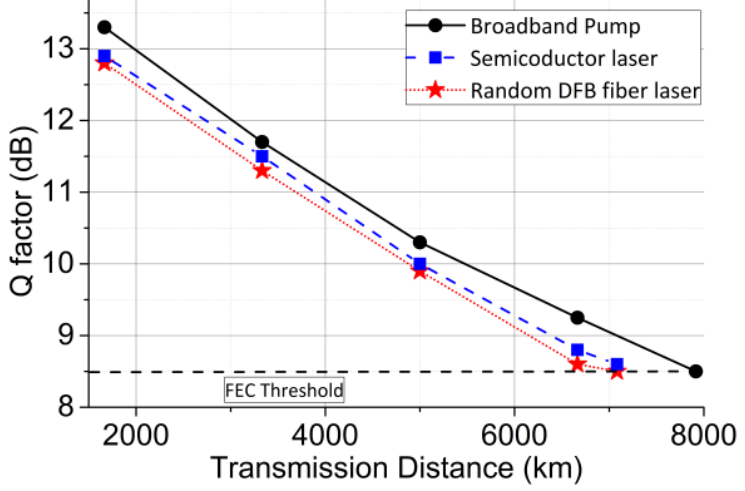

Fig. 7. Measured $1545.3 \mathrm{~nm}$ central channel $\mathrm{Q}$ factors versus transmission distances using broadband pump, semiconductor laser, or random DFB fiber laser in dual order BW pumping configurations.
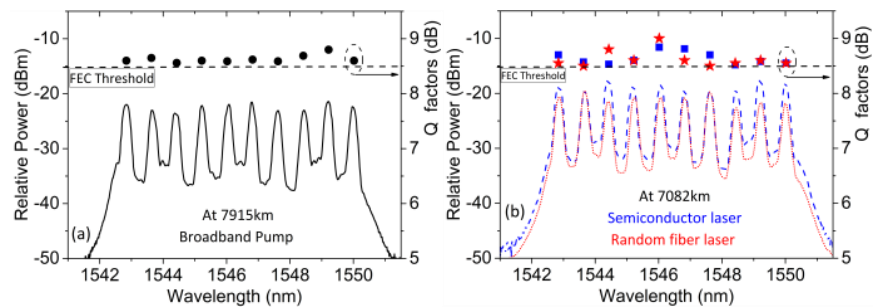

Fig. 8. Received spectra and $\mathrm{Q}$ factors for all the ten channels at its maximum reach. (a). broadband pump; (b). semiconductor laser/random DFB fiber laser;

Figure 7 showed the maximum reach of the central channel at $1545.3 \mathrm{~nm}$ with the optimum launch power, using different first order pumps in dual order DRA. As expected from Fig. 6, using the broadband pump gave the longest transmission distance of $7915 \mathrm{~km}$, due to the mitigation of RIN transfer. Both the semiconductor laser and random fiber laser configurations gave the same reach of $7082 \mathrm{~km}$, but the Q factor at $7082 \mathrm{~km}$ in the semiconductor laser configuration was slightly better as the first order semiconductor laser showed lower RIN. Fig. 8 shows the received spectra and $\mathrm{Q}$ factors for all the ten channels at their maximum transmission distances. All the measured $\mathrm{Q}$ factors were above the FEC threshold $(8.5 \mathrm{~dB})$ which is equivalent to the BER of $3.8 \times 10^{-3}$.

\section{CONCLUSION}

We have demonstrated that, for the first time, using only a 20 $\mathrm{mW}$ incoherent broadband first order pump in a dual order backward-pumped DRA can mitigate the RIN transfer from second order backward-propagated pumps to the signal. This allows a $\sim 12 \%$ reach enhancement in repeatered transmission systems, compared with dual order DRA schemes using low RIN semiconductor laser and cost-effective FBG assisted random fiber laser. Such broadband pump has the potential of providing flatter and wider gain spectrum which can effectively reduce the number of pumps for the broadband transmission system.

\section{REFERENCES}

[1] W. S. Pelouch, "Raman Amplification: An Enabling Technology for Long-Haul Coherent Transmission Systems," J. Lightwave Technol., vol. 34, no. 1, pp. 6-19, Jan.1, 12016

[2] L. Galdino et al., "Amplification Schemes and Multi-Channel DBP for Unrepeatered Transmission," J. Lightwave Technol, vol. 34, no. 9, pp. 2221-2227, May.1, 12016.

[3] J. D. Ania-Castanon, "Quasi-lossless transmission using second-order Raman amplification and fibre Bragg gratings," Opt. Express, vol. 12, no. 19, pp. 4372-4377, 2004

[4] M. Tan et al., "Evaluation of 100G DP-QPSK long-haul transmission performance using second order co-pumped Raman laser based amplification," Opt. Express, vol. 23, no. 17, pp. 22181-22189, 2015.

[5] L. Xu et al., "Experimental Verification of Relative Phase Noise in Raman Amplified Coherent Optical Communication System," J. Lightwave Technol., vol. 34, no. 16, pp. 3711-3716, 2016.

[6] S. Burtsev et al., "Pump-to-Signal Cross-Polarization Scattering in Coherent Dual-Polarized Systems with Forward Raman Amplification," Proc. OFC, Anaheim, CA, USA, 2016, paper Th2A.48.

[7] C. R. S. Fludger et al., "Pump to signal RIN transfer in Raman fiber amplifiers," J. Lightwave Technol., vol.19, no.8, pp. 1140-1148, 2001.

[8] M. Tan et al., "Transmission performance improvement using random DFB fiber laser based Raman amplification and bidirectional secondorder pumping," Opt. Express, vol. 24, no. 3, pp. 2215-2221, 2016.

[9] M. Morimoto et al., "Co-Propagating Dual-Order Distributed Raman Amplifier Utilizing Incoherent Pumping," IEEE Photon. Technol. Lett., vol. 29, no. 7, pp. 567-570, 2017.

[10] M. Tan et al., "RIN Mitigation and Transmission Performance Enhancement With Forward Broadband Pump," IEEE Photon. Technol. Lett, vol. 30, no. 3, pp. 254-257, Feb.1, 12018.

[11] S. B. Papernyi et al., "Third-order cascaded Raman amplification," in Proc. OFC, Anaheim, CA, USA, 2002, pp. 1-3, paper FB4.

[12] G. Rizzelli et al., "Impact of input FBG reflectivity and forward pump power on RIN transfer in ultralong Raman laser amplifiers," Opt. Express, vol. 24, no. 25, pp. 29170-29175, 2016

[13] W. L. Zhang et al., "Low threshold 2nd-order random lasing of a fiber laser with a half-opened cavity," Opt. Express, vol. 20, no.13, pp. 14400$14405,2012$.

[14] Y. Wang et al., "A Distributed Raman Amplifier Based on the BackwardPumped Random Fiber Laser," IEEE Photon. Technol. Lett., vol. 30, no. 2, pp. 173-176, Jan.15, 152018.

[15] S. A. Babin et al., "Random fiber laser directly pumped by a high-power laser diode," Opt. Lett., vol.38, no. 17, pp.3301-3303, 2013.

[16] G. Saavedra et al., "Experimental Analysis of Nonlinear Impairments in Fibre Optic Transmission Systems up to 7.3 THz," J. Lightwave Technol., vol. 35, no. 21, pp. 4809-4816, Nov.1, 12017.

[17] A. H. Gnack et al., "Demonstration of counter-propagating Raman pump placed near Signal-channel wavelength," IEEE Photon. Technol. Lett., vol. 29, no. 1, pp. 154-157, Jan.1, 2017.

[18] D. V. Churkin et al., "Raman Fiber Lasers with a Random Distributed Feedback Based on Rayleigh Scattering," Physical Review A, vol. 82, no. 3, pp. 033828, 2010.

[19] S. K. Turitsyn et al., "Random distributed feedback fibre lasers," Physics Reports, vol. 542, no. 10, pp. 133-193, Sept. 10, 2014

[20] K. Keita et al., "Relative intensity noise transfer of large-bandwidth pump lasers in Raman fiber amplifiers," J. Opt. Soc. Am. B., vol. 23, no. 12, pp. 2479-2485, 2006 